\title{
Análisis de Las Capacidades Emprendedoras Potenciales y Efectivas en Alumnos de Centros de Educación Superior
}

\author{
Ricardo Cabana-Villca', Ivan Cortes-Castillo², Diego Plaza-Pasten, Mauricio Castillo-Vergara ${ }^{4}$, \\ Alejandro Alvarez-Marin ${ }^{5}$
}

\begin{abstract}
There were identified four factors that determine the enterprising potential capacity of a pupil belonging to higher education centers (attributes of the entrepreneur, interpersonal capacities, capacities opposite to the risk and attitude of the entrepreneur). On having organized into a hierarchy using the AHP method, it concluded the vectors of priority giving a major importance for attitude and attributes of the entrepreneur, which as a whole represent $76 \%$. The index of enterprising potential capacity was $74 \%$ and the percentage of pupils with effective enterprising capacity was $16 \%$. This gap of development of enterprising capacity represents a strategic challenge for each higher education centers, to contribute to its value and in addition to the local, regional and national development. There proposes a model of analysis of the enterprising capacities, who would allow significant achievements in the formation of effective enterprising capacities.

Se identificaron cuatro factores que determinan la capacidad emprendedora potencial de un alumno perteneciente a centros de educación superior (atributos del emprendedor, capacidades interpersonales, capacidades frente al riesgo y actitud del emprendedor). Al jerarquizar empleando el método AHP, se concluyó los vectores de prioridad dando una mayor importancia para actitud y atributos del emprendedor, que en conjunto representan el 76\%. El índice de capacidad emprendedora potencial fue de un $74 \%$ y el porcentaje de alumnos con capacidad emprendedora efectiva fue de un $16 \%$. Esta brecha de desarrollo de capacidad emprendedora, representa un desafío estratégico para cada uno de los centros de educación superior, para contribuir a su valor y complementariamente al desarrollo local, regional y nacional. Se propone un modelo de análisis de las capacidades emprendedoras, que permitiría logros significativos en la formación de capacidades emprendedoras efectivas.
\end{abstract}

Keywords: enterprising capacity; higher education; entrepreneurship; entrepreneurial. capacidad emprendedora; centros de educación superior; emprendimiento.

Departamento de Ingeniería Industrial, Universidad de La Serena, Benavente 980, La Serena, I720I70, Chile. Phone: 56-5I-204262. email: 'rcabana@userena.cl, ${ }^{2}$ ivn.cortes@gmail.com, ${ }^{3} \mathrm{db} . p l a z a @ g m a i l . c o m,{ }^{4}$ mhcastillo@userena.cl, ${ }^{5}$ aalvarez@userena.cl.

ISSN: 07 I8-2724. (http://www.jotmi.org)

Journal of Technology Management \& Innovation (C) Universidad Alberto Hurtado, Facultad de Economía y Negocios. 


\section{Introducción}

El emprendedor es un individuo líder, previsor, tomador de riesgos, evaluador de proyectos, y que moviliza recursos desde una zona de bajo rendimiento a una de alta productividad (Burnett, 2000). También puede ser considerado como una persona dinámica y fuera de lo común que promueve nuevas combinaciones o innovaciones, que con sus acciones causa inestabilidad en el mercado (Schumpeter, 1942). El emprendedor puede ser definido como aquel empresario que es innovador y no se debe caer en la confusión de creer que cualquier negocio pequeño y nuevo es un emprendimiento, y quien lo lleva a cabo un emprendedor (Formichella, 2004). El estudio del emprendedor se puede analizar bajo 3 perspectivas ( Krauss, 2005).
Primero bajo una perspectiva psicológica, en el cual se explican 3 teorías (Cross y Travaglione, 2003). La primera está relacionada con la motivación (Mc Clelland, 196I). La segunda está relacionada con la teoría de la personalidad o del rasgo (Busenitz, 1999), (Mc Carthy, 2003), (Stewart y Roth, 200I) y la última se denomina teoría cognitiva (Neck et. al., 1999).

Segundo, bajo una perspectiva demográfica, la cual está asociada principalmente a los antecedentes familiares, o sea determinar la existencia de familiares emprendedores, estado civil, edad, nivel educacional, experiencia laboral, status socioeconómico, entre otros, variables que condicionan las capacidades emprendedoras (Cano, García, y Gea, 2003).

\begin{tabular}{|c|c|}
\hline Variable & Autores \\
\hline Identificación de oportunidades & $\begin{array}{l}\text { (Timmos, I985), (Amorós y Poblete Cazenave, 2010), (Palacios, } \\
\text { 1999), (Garzón, 2004). }\end{array}$ \\
\hline Proactividad & (Filion y Gilles, 1996), (Estudio Hay Group, 2000). \\
\hline Creatividad & $\begin{array}{l}\text { (Filion y Gilles, I996), (Bilbao y Pachano, 2002), (Loli et. al., 2010), } \\
\text { (Alcaraz Rodriguez, 2004), (Alfaro y Gómez, 1999), (Garzón, } \\
\text { 2004). }\end{array}$ \\
\hline Visión & $\begin{array}{l}\text { (Filion y Gilles, I996), (Loli et. al., 20 I0), (Hernan Gomez Bara- } \\
\text { hona, et. al., 2005). }\end{array}$ \\
\hline Habilidades Administrativas & (Gonzalés y Zuñiga, 20II). \\
\hline Trabajo en Equipo & $\begin{array}{l}\text { (Filion y Gilles, I996), (Bilbao y Pachano, 2002), (Gonzalés y Zu- } \\
\text { ñiga, 20 I I), (Palacios, 1999). }\end{array}$ \\
\hline Participación & $\begin{array}{l}\text { (Filion y Gilles, 1996), (Alcaraz Rodriguez, 2004), (Palacios, 1999), } \\
\text { (Estudio Hay Group, 2000). }\end{array}$ \\
\hline Comunicación & $\begin{array}{l}\text { (Filion y Gilles, 1996), (Gonzalés y Zuñiga, 201 I), (Palacios, I999), } \\
\text { (Cantillon, I 155). }\end{array}$ \\
\hline Liderazgo & $\begin{array}{l}\text { (Filion y Gilles, I 996), (Estudio Hay Group, 2000), (Bilbao y Pacha- } \\
\text { no, 2002), (Loli et. al., 2010), (Alcaraz Rodriguez, 2004). }\end{array}$ \\
\hline Adaptabilidad & $\begin{array}{l}\text { (Filion y Gilles, 1996), (Loli et. al., 2010), (Timmos, I985), (Alcaraz } \\
\text { Rodriguez, 2004). }\end{array}$ \\
\hline Sentido de Riesgo & $\begin{array}{l}\text { (Filion y Gilles, I996), (Palacios, 1999), (Bilbao y Pachano, 2002), } \\
\text { (Loli et. al., 20 I0), (Timmos, I 985), (Vander Werf y C. Brush, 1989), } \\
\text { (Alcaraz Rodriguez, 2004). }\end{array}$ \\
\hline Confianza & $\begin{array}{l}\text { (Filion y Gilles, I 996), (Estudio Hay Group, 2000), (Bilbao y Pach- } \\
\text { ano, 2002), (Loli et. al., 2010), (Timmos, 1985), (Alcaraz Rodriguez, } \\
\text { 2004). }\end{array}$ \\
\hline Constancia & $\begin{array}{l}\text { (Filion y Gilles, I996), (Bilbao y Pachano, 2002), (Timmos, I985), } \\
\text { (Alcaraz Rodriguez, 2004). }\end{array}$ \\
\hline Compromiso & $\begin{array}{l}\text { (Filion y Gilles, 1996), (Estudio Hay Group, 2000), (Palacios, 1999), } \\
\text { (Loli et. al., 2010), (Timmos, 1985). }\end{array}$ \\
\hline Pasión & (Bilbao y Pachano, 2002), (Timmos, 1985). \\
\hline Motivación & $\begin{array}{l}\text { (Timmos, 1985), (Vander Werf y C. Brush, 1989), (Filion y Gilles, } \\
\text { 1996), (Palacios, 1999). }\end{array}$ \\
\hline
\end{tabular}

TABLA I Variables relacionadas con las perspectivas actitudinal y psicológica

ISSN: 07 I8-2724. (http://www.jotmi.org)

Journal of Technology Management \& Innovation (C) Universidad Alberto Hurtado, Facultad de Economía y Negocios. 
Tercero, bajo una perspectiva actitudinal, ya que la actitud es la predisposición a responder a un determinado acontecimiento $y$ es la componente principal del perfil de un emprendedor, además de ser modificable tanto por el paso del tiempo así como por la interacción con el entorno (Robinson, |991).

Considerando las 3 perspectivas antes mencionadas, se identifican 16 variables a nivel individual que están principalmente relacionadas con las perspectivas actitudinal $y$ psicológica (TABLA I).

\section{Emprendimiento y los Centros de Educación Superior (CES)}

A nivel nacional prácticamente la mitad de los emprendedores no tiene niveles educativos más allá de la educación obligatoria (GEM Reporte Nacional de Chile, 20I0).

Según el GEM de la región de Coquimbo (2009-2010) la edad promedio de las personas que emprenden en su etapa inicial es de 36 años y 46 años para los empresarios establecidos, mientras que el $18 \%$ de los emprendedores en esta etapa tiene estudios superiores.

En cuanto a los centros de educación superior, la educación tiene una responsabilidad central en identificar $y$ fomentar a las personas que pueden ser emprendedores (Hatten, 1993), por lo tanto cada vez es mayor el consenso que existe, sobre el rol de la universidad como formadora de emprendedores. Para las universidades el hecho de formar emprendedores es considerado un motor de desarrollo (Laukkanen, 2000).

De acuerdo con lo anterior es necesario promover el emprendimiento en los CES, dado que cuanto más intensiva sea la enseñanza del emprendimiento en las instituciones de educación terciaria, más probabilidades habrá de que los estudiantes hagan el esfuerzo por empezar un nuevo negocio $y$, de esa forma, contribuir al desarrollo de la economía (Alvarez y Jung, 2004).

El "Global Entrepreneurship Monitor" (GEM), es un informe anual que muestra el estado del emprendimiento en Chile y las principales barreras que han impedido su desarrollo. Se inició en el año 2002 y es parte del proyecto GEM Mundial del cual han participado más de 60 países.

De acuerdo a la metodología del GEM, los emprendimientos en etapa inicial (TEA) se dividen en dos categorías: I) los emprendimientos nacientes, es decir aquellas personas que están en los tres primeros meses de su emprendimiento $y, 2$ ) los propietarios de nuevos negocios, que son aquellos que ya arrancaron el emprendimiento y que tiene una antigüedad mayor a los tres meses y menor a los 3.5 años.
Por esta razón, el desafío que existe es cómo fomentar continuamente el espíritu emprendedor en los estudiantes. Un estudio identifica las variables que alientan la intención emprendedora en este grupo objetivo, generando un modelo que incorpora el Clima de Emprendimiento en la Universidad, como factor en esta intención (Krueger y Brazeal, 1994). Se puede definir clima de emprendimiento como el conjunto de condiciones y circunstancias que rodean un lugar específico y que afectan directa o indirectamente las intenciones emprendedoras de las personas que están vinculadas a este espacio. El clima de emprendimiento debe ser visto desde una óptica sistémica en la cual inciden factores de diversa índole, tanto formales como informales (Morales Parragué, 2009).

Un modelo complementario de emprendimiento considera tres factores: Formación del emprendedor, promoción del valor del emprendimiento e innovación y desarrollo de un ecosistema de apoyo. Para maximizar la eficiencia de este modelo, este proceso debe llevarse a cabo de manera integrada y acelerada para lograr ejemplos exitosos que autoalimenten el movimiento (Vicens y Grullón, 20I I).

\section{Materiales y métodos}

El propósito de esta investigación es determinar y analizar las capacidades emprendedoras potenciales y efectivas en alumnos de centros de educación superior. Para su evaluación se utilizaron las 16 variables identificadas, las cuales fueron agrupadas en 4 factores para su jerarquización (TABLA 2).

\section{Método de jerarquización e importancia de factores y variables}

Se utilizó el método de decisión multicriterio denominado "The Analytic Hierarchy Process" (AHP), desarrollado por Thomas Saaty. Este método consiste en construir matrices a partir de la comparación por pares de elementos, utilizando elementos del algebra matricial para establecer los vectores prioridad asociado a cada factor $y$ variable. Este método es ampliamente aplicado en la solución de una gran variedad de problemas (Osorio Gómez y Orejuela Cabrera, 2008).

Para esta investigación se utilizó un panel de expertos, pues implica gran credibilidad en las conclusiones, ahorros de tiempo y costos del proceso de evaluación (Ayuga Téllez et. al., 2012). El panel de expertos se constituyó por 9 profesionales ligados al emprendimiento en el ámbito público y/o privado, 2 académicos expertos en emprendimiento, 2 directores de prestigiosas consultoras a nivel nacional y 5 directores de organismos públicos relacionados con el emprendimiento. 
Los expertos tuvieron que responder 2 cuestionarios. En el primero, los expertos jerarquizaron los factores y variables, desde el más importante al menos importante. En el segundo cuestionario evaluaron el grado de importancia de factores y variables realizando comparaciones duales, utilizando la escala de importancia propuesta por Saaty.

Los resultados obtenidos a partir de la metodología AHP muestran que ninguna matriz superó la razón de inconsistencia (RI) máxima permitida del $10 \%$, por lo tanto se aceptan los valores de la matrices como consistentes y por consiguiente los vectores prioridad de cada factor $y$ variable (TABLA 3 y TABLA 4).

\section{Instrumento evaluativo}

El instrumento final constó de 50 ítems el cual estuvo compuesto en un $95 \%$ de preguntas politómicas con una escala de tipo Likert y las restantes preguntas fueron del tipo de respuestas dicotómicas.

Para el análisis de la consistencia interna se utilizó el cálculo de los coeficientes de correlación de Pearson ítem-total, y para el análisis de la fiabilidad del instrumento se calculó el coeficiente de Alpha de Cronbach, como lo realizan diferentes autores (López Pumar et. al., 20II), (Granado de la Orden et. al., 2007), (Bautista García y Mateos Moreno, 20I2).

Los coeficientes de correlación de Pearson, pueden oscilar entre valores de $+I$ a $-I$, dentro de los cuales los valores menores a 0,30 deben ser desechados o reformulados (Lucero y Meza, 2002). La depuración de los ítems tiene como finalidad aumentar el coeficiente de fiabilidad del instrumento. Por otro lado, el Alpha de Cronbach se considera aceptable sobre 0,65 y se califica como excelente cuando es superior a 0,9 (Mallery, 1995) (Köttner Jan, 2010).

Para estos fines se aplicó un instrumento preliminar o piloto que constaba de 59 ítems a una muestra de 120 alumnos, ya que para realizar un análisis de fiabilidad el número de sujetos deber ser el doble del número de ítems (Morales Vallejos, 20I2).

Para el análisis estadístico de los resultados se utilizó el programa SPSS \{SPSS (Statistical Package for the Social Sciences), programa para el análisis y tratamiento estadístico\}y Microsoft Excel 2010.

La confiabilidad del instrumento final con 50 ítems aumentó a un 0,916 lo que indica un excelente nivel de confiabilidad. En cuanto a la confiabilidad para cada factor fue de 0,756 para la Actitud del Emprendedor, 0,834 Atributos del Emprendedor, 0,660 Capacidad del Emprendedor y 0,789 Capacidades Interpersonales del Emprendedor.

\section{Muestra}

El tipo de muestreo utilizado fue probabilístico y de tipo estratificado en alumnos que cursaban sus últimos semestres, seleccionándose 94 carreras relacionadas con el desarrollo de emprendimientos en 12 CES de las comunas de La Serena y Coquimbo (universidades tradicionales, universidades privadas, institutos de formación técnica y centros de formación técnica) divididas en 6 estratos a los cuales se les llamó áreas de conocimientos, estas fueron: I) Diseño y comunicaciones, 2) Ciencias, 3) Ciencias sociales y económicas, 4) Hotelería, turismos y gastronomía, 5) Informática y telecomunicaciones, 6) Ingeniería.

La muestra fue de 389 alumnos cumpliendo con un nivel de confianza de un $95 \%$ y un error muestral de $5 \%$.

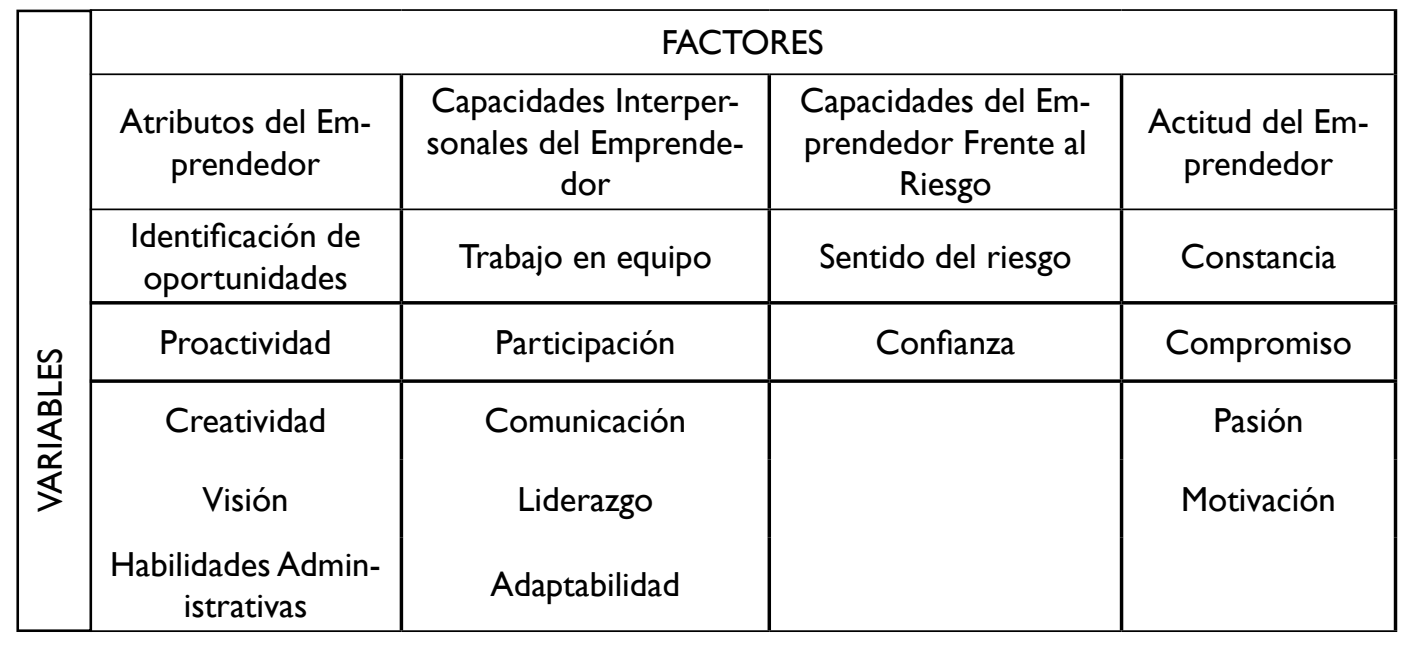

TABLA 2 Factores y variables críticas

ISSN: 07 I8-2724. (http://www.jotmi.org)

Journal of Technology Management \& Innovation (c) Universidad Alberto Hurtado, Facultad de Economía y Negocios. 


\begin{tabular}{|c|c|c|c|c||c|}
\hline Factor & $\begin{array}{c}\text { Actitud del } \\
\text { Emprendedor }\end{array}$ & $\begin{array}{c}\text { Atributo del } \\
\text { Emprendedor }\end{array}$ & $\begin{array}{c}\text { Capacidad del Em- } \\
\text { prendedor Frente } \\
\text { al Riesgo }\end{array}$ & $\begin{array}{c}\text { Capacidad Interper- } \\
\text { sonal Emprendedor } \\
(1)\end{array}$ & $\begin{array}{c}\text { Vector } \\
\text { Prioridad }\end{array}$ \\
\hline$($ I) & $\mathrm{I}$ & $2,5 \mathrm{I}$ & $3,6 \mathrm{I}$ & 3,62 & $47 \%$ \\
\hline$(2)$ & 0,40 & $\mathrm{I}$ & 2,90 & 4,27 & $29 \%$ \\
\hline$(3)$ & 0,28 & 0,34 & $\mathrm{I}$ & 3,23 & $16 \%$ \\
\hline$(4)$ & 0,28 & 0,23 & $0,3 \mathrm{I}$ & $\mathrm{I}$ & $8 \%$ \\
\hline
\end{tabular}

Razón Inconsistencia (RI) 8,8\%

TABLA 3 Vector prioridad para cada factor

\begin{tabular}{|l|c|l|c|}
\hline $\begin{array}{c}\text { ACTITUD DEL EMPRENDE- } \\
\text { DOR }\end{array}$ & $\begin{array}{l}\text { VECTOR PRI- } \\
\text { ORIDAD }\end{array}$ & \multicolumn{1}{|c|}{$\begin{array}{c}\text { ATRIBUTO DEL EM- } \\
\text { PRENDEDOR }\end{array}$} & $\begin{array}{c}\text { VECTOR } \\
\text { PRIORIDAD }\end{array}$ \\
\hline Constancia & $38 \%$ & $\begin{array}{l}\text { Identificación de Oportuni- } \\
\text { dades }\end{array}$ & $39 \%$ \\
\hline Motivación & $27 \%$ & Proactividad & $24 \%$ \\
\hline Pasión & $18 \%$ & Creatividad & $21 \%$ \\
\hline Compromiso & $17 \%$ & Visión & $10 \%$ \\
\hline & & Habilidades Administrativas & $6 \%$ \\
\hline Razón Inconsistencia & $4,8 \%$ & Razón Inconsistencia & $4,9 \%$ \\
\hline $\begin{array}{c}\text { CAPACIDAD DEL EM- } \\
\text { PRENDEDOR FRENTE AL } \\
\text { RIESGO }\end{array}$ & VECTOR PRI- & $\begin{array}{l}\text { CAPACIDAD INTERPER- } \\
\text { SONAL DEL EMPRENDE- }\end{array}$ & $\begin{array}{c}\text { VECTOR } \\
\text { PRIORIDAD }\end{array}$ \\
\hline Confianza & $80 \%$ & Liderazgo & $41 \%$ \\
\hline Sentido del Riesgo & $20 \%$ & Trabajo en Equipo & $24 \%$ \\
\hline & & Adaptabilidad & $14 \%$ \\
\hline & & Comunicación & $13 \%$ \\
\hline & & Participación & $8 \%$ \\
\hline & $0 \%$ & Razón Inconsistencia & $7,0 \%$ \\
\hline Razón Inconsistencia & & & \\
\hline
\end{tabular}

TABLA 4 Vector prioridad para cada variable por factor

\section{Capacidad Emprendedora Potencial y Efectiva}

La capacidad emprendedora potencial se medirá a través del índice de Capacidad Emprendedora Potencial (ICEP) el cual se construirá utilizando el promedio ponderado entre los resultados de los factores y los vectores prioridad determinados por los expertos.
FI:Actitud del Emprendedor.

F2:Atributo del Emprendedor.

F3: Capacidad del Emprendedor Frente al Riesgo.

F4: Capacidades Interpersonales del Emprendedor.

Pr:Vector Prioridad.

$\mathrm{n}$ :Valor máximo de la Escala Likert (5).

$$
I C E P=\frac{F 1 * P r_{1}+F 2 * \mathrm{Pr}_{2}+F 3 * \mathrm{Pr}_{3}+F 4 * \mathrm{Pr}_{4}}{n} \times 100 \text { (\%) }
$$




\begin{tabular}{|c|c|c|c|c|c|c|}
\hline \multirow[b]{2}{*}{$\begin{array}{c}\text { Área de } \\
\text { conocimiento }\end{array}$} & \multirow[b]{2}{*}{$\begin{array}{c}\text { Porcentaje } \\
\text { de la Mues- } \\
\text { tra }\end{array}$} & \multicolumn{2}{|c|}{ Género } & \multicolumn{3}{|c|}{ Tipo de CES } \\
\hline & & $\begin{array}{l}\text { Porcentaje } \\
\text { Femenino }\end{array}$ & $\begin{array}{l}\text { Porcentaje } \\
\text { Masculino }\end{array}$ & $\begin{array}{c}\text { Porcentaje } \\
\text { Universidades } \\
\text { del CRUCH }\end{array}$ & $\begin{array}{c}\text { Porcentaje } \\
\text { Universidades } \\
\text { Privadas }\end{array}$ & $\begin{array}{c}\text { Porcentaje } \\
\text { CFT+IP }\end{array}$ \\
\hline Ingeniería & 34 & 34 & 66 & 40 & 15 & 45 \\
\hline $\begin{array}{l}\text { Ciencias Sociales y } \\
\text { Económicas }\end{array}$ & 37 & 57 & 43 & 10 & 32 & 58 \\
\hline $\begin{array}{l}\text { Informática y Tel- } \\
\text { ecomunicaciones }\end{array}$ & 15 & 24 & 76 & 9 & 9 & 82 \\
\hline $\begin{array}{l}\text { Hotelería,Turismo } \\
\text { y Gastronomía }\end{array}$ & 6 & 76 & 24 & 20 & 0 & 80 \\
\hline $\begin{array}{l}\text { Diseño y Comuni- } \\
\text { caciones }\end{array}$ & 4 & 60 & 40 & 67 & 33 & 0 \\
\hline Ciencias & 4 & 47 & 53 & 67 & 0 & 33 \\
\hline
\end{tabular}

TABLA 5 Descripción de la muestra

La capacidad emprendedora efectiva se medirá a través del porcentaje de alumnos que han materializado una oportunidad de negocio, lo que da como resultado la ejecución o implementación de un emprendimiento.

\section{Discusión y resultados}

Los resultados arrojaron que el índice de capacidad emprendedora potencial (ICEP) promedio es del 74\% (género masculino $75 \%$, mientras que para el género femenino resultó ser de un 74\%) que representa un nivel de capacidad emprendedora potencial avanzada, lo que implica que el proceso de formación, los recursos de enseñanza y aprendizaje, y la cultura organizacional pro-emprendimiento del CES ha provocado una evolución normal de la capacidad emprendedora del alumno. Este alumno está en condiciones para implementar intraemprendimientos y/o desarrollar las oportunidades de negocios identificadas durante el pregrado ya sea de manera individual o siendo parte de un equipo.

Complementariamente el porcentaje de alumnos de la muestra con capacidad emprendedora efectiva promedio es de un $16 \%$, compuesto por un $13 \%$ de género femenino y un $19 \%$ masculino.

Lo anterior refleja la brecha en el desarrollo de capacidades emprendedoras en alumnos de pregrado (Brecha DCE), por área del conocimiento (FIGURA I) y por tipo de CES (GRÁFICO I).

\section{Análisis de factores y variables}

Todos los factores están dentro del tipo de capacidad de emprendimiento potencial avanzada, rango según escala propuesta 60 - 80\% (GRÁFICO 2).
Por otra parte las variables que explican estos factores también están dentro del tipo de capacidad emprendedora potencial avanzada (GRÁFICO 3), siendo las variables peor evaluadas: la visión, participación y la identificación de oportunidades. Cabe destacar la importancia de esta última por su incidencia en la capacidad de emprendimiento efectiva, es decir, en la ejecución de una idea u oportunidad de negocio.

\section{Conclusiones}

Es importante mencionar que los alumnos que desarrollan o ejecutan emprendimientos, tienen un índice de capacidad emprendedora potencial de un $78 \%$ y los alumnos que no lo hacen tienen un índice de un $74 \%$, por lo tanto no existe una gran diferencia en cuanto a las capacidades emprendedoras potenciales de los alumnos que desarrollan oportunidades de negocio y los que no lo hacen, situación que ya ha sido planteada por otros autores (Albacete Saez et.al., 2008-10). Por lo tanto lo relevante está en el costo de oportunidad asociado a la brecha en el desarrollo de capacidades emprendedoras, dado el alto número de alumnos que no contribuye a la cadena de valor de cada CES, y en la necesidad que mayor porcentaje de alumnos evolucionen desde un nivel intermedio a nivel avanzado y desde allí al nivel de capacidad emprendedora potencial consolidada.

El que exista una gran brecha entre las capacidades emprendedoras potenciales y efectiva, produce un alto costo de oportunidad en los CES, al no internalizar beneficios que podrían generar sus alumnos con la ejecución de sus emprendimientos e intraemprendimientos durante el pregrado, incrementando el valor agregado de cada CES al; mejorar el proceso de enseñanza y aprendizaje, la calidad de servicio administrativo y educacional, incrementar la contribución

ISSN: 07 I8-2724. (http://www.jotmi.org) 


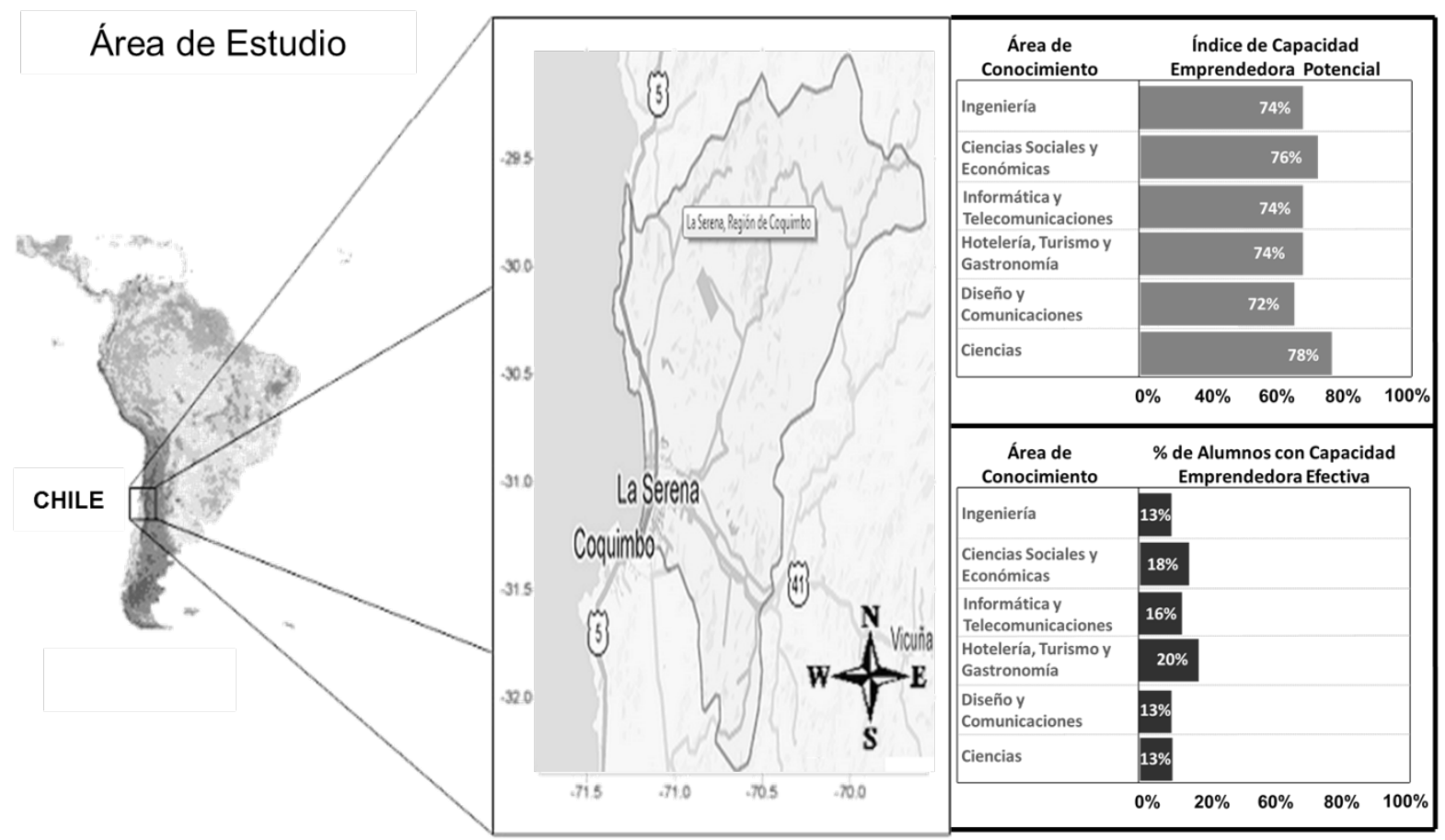

FIGURA I Brechas DCE por Área de Conocimiento

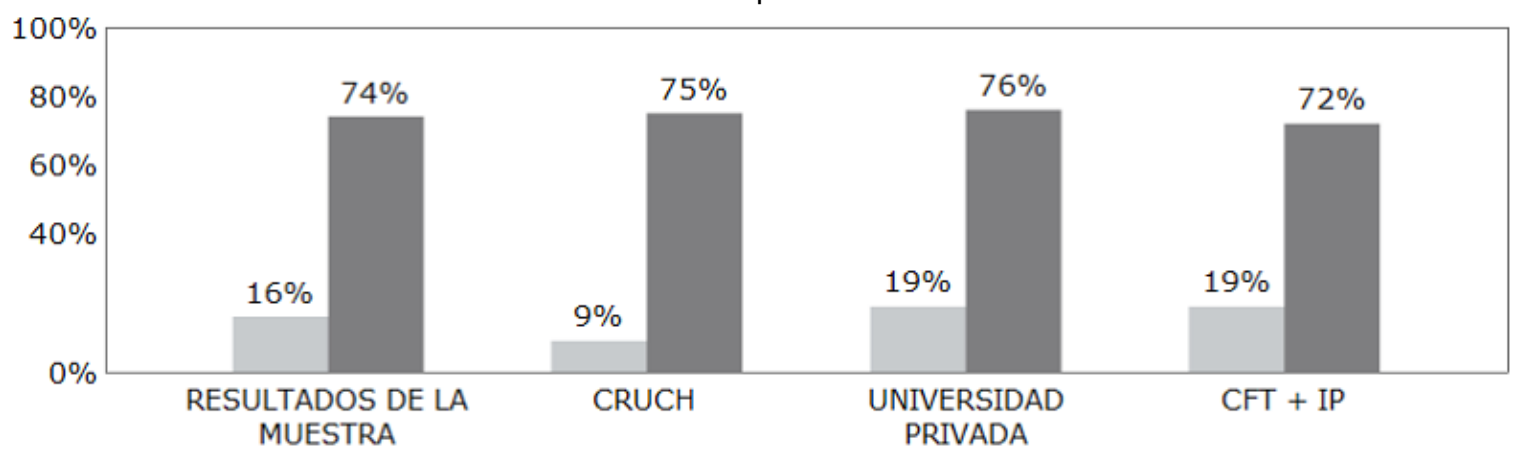

Ill Porcentaje de alumnos con Capacidad Emprendedora Efectiva

Illa Índice de Capacidad Emprendedora Potencial

GRÁFICO I Brechas DCE por Tipo de CES

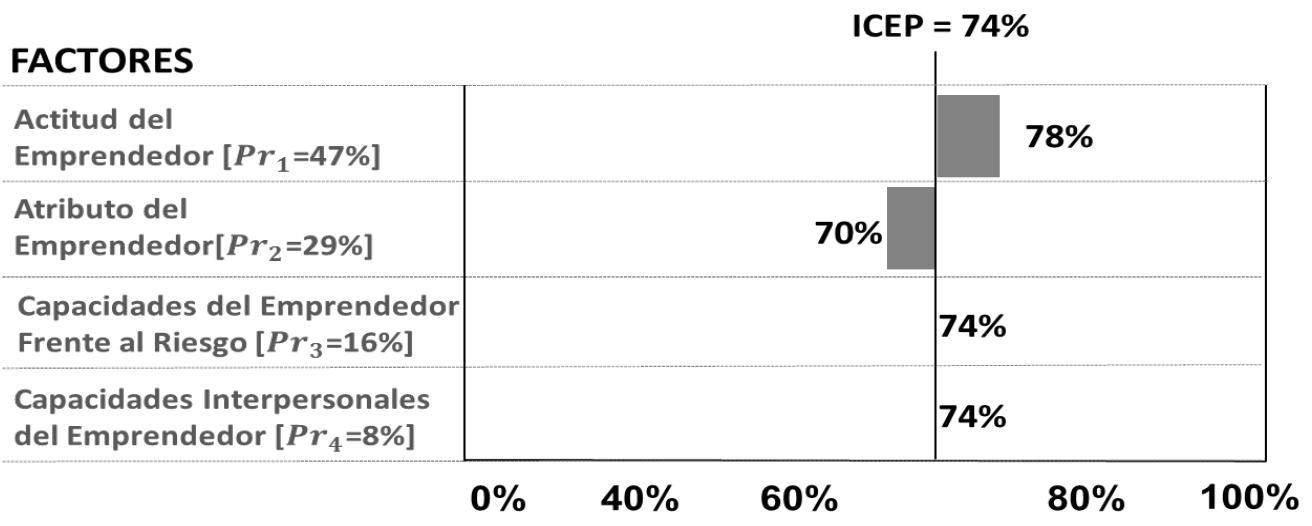

GRÁFICO 2 Resultados por Factores

ISSN: 07 I8-2724. (http://www.jotmi.org)

Journal of Technology Management \& Innovation (c) Universidad Alberto Hurtado, Facultad de Economía y Negocios. 
al desarrollo local y nacional, y como consecuencia lograr mejoras en la empleabilidad del alumno, lo cual generaría un impacto positivo en el valor de la carrera y de cada CES. La evolución de capacidad emprendedora potencial a efectiva, depende del proceso de formación del emprendedor, la promoción del valor del emprendimiento e innovación y del desarrollo de un ecosistema de apoyo. Durante la investigación se identifican un ecosistema transformador interno y otro externo. El primero está directamente relacionado con los CES y está compuesto por 4 agentes transformadores los cuales son; académicos, recursos de formación, estrategias de enseñanza y redes internas de alumnos. El segundo ecosistema está compuesto por 3 agentes transformadores los cuales son: apoyo gubernamental y privado, padres-familia y finalmente la empresa.
El modelo que planteamos considera además la existencia de un macro entorno que está compuesto por: entornos económico, social y cultural, legal y medio ambiental y finalmente un entorno tecnológico (FIGURA 2).

Por lo tanto, el objetivo de los directivos de cada CES y de los responsables de la implementación de sus respectivos modelos educativos, es lograr que tanto los agentes internos como externos, influyan sistémicamente en el alumno permitiéndole evolucionar hacia una capacidad emprendedora efectiva, pues en ese escenario se demuestra el valor de los CES en su contribución estratégica en el alumno, la comuna, la región y el país.

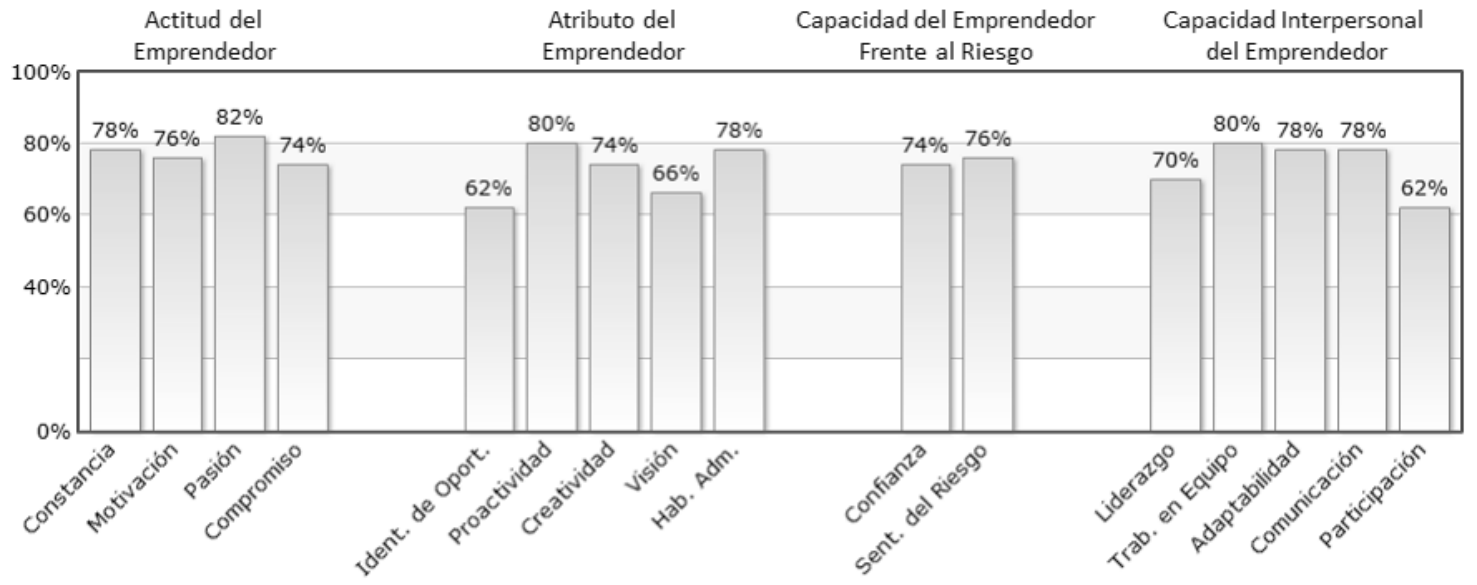

GRÁFICO 3 Resultados por Variables

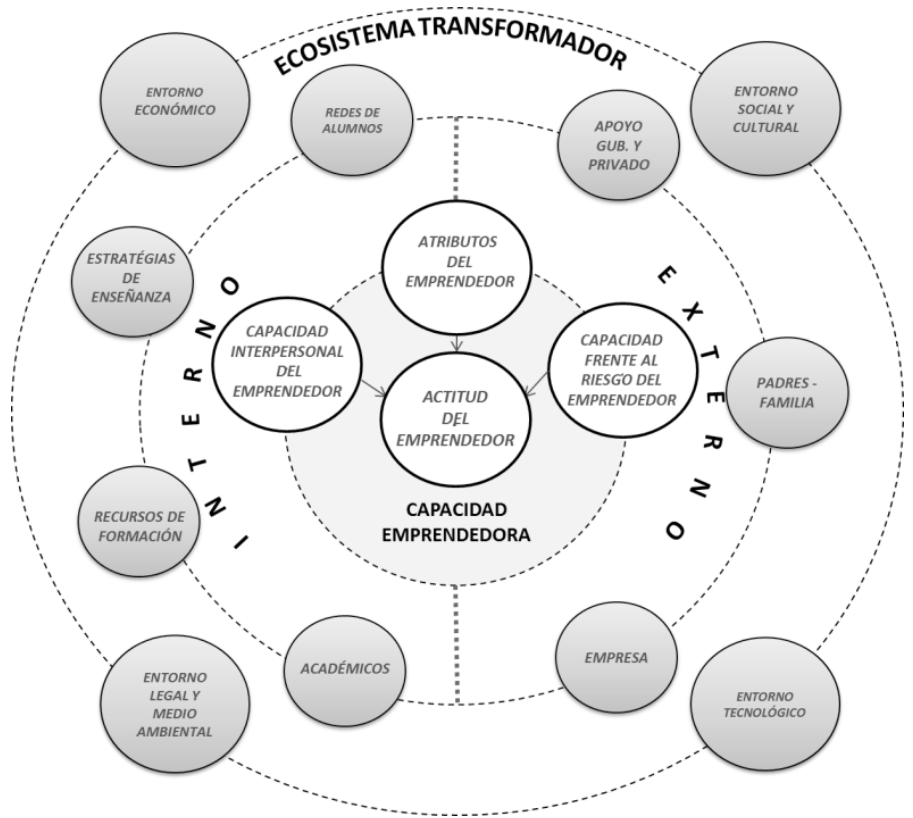

FIGURA 2 Modelos de análisis de las capacidades emprendedoras. Fuente: Elaboración propia.

ISSN: 07 I8-2724. (http://www.jotmi.org)

Journal of Technology Management \& Innovation (c) Universidad Alberto Hurtado, Facultad de Economía y Negocios. 
Si las instituciones educacionales logran la formación de una alta capacidad de emprendimiento efectivo, los alumnos se constituirían en potenciales inversionistas que con mayor probabilidad implementarían empresas con innovación competitiva y valor económico sustentable, es decir, empresas donde los procesos de innovación y emprendimiento se potencian, siendo esto la base de su diferenciación y posicionamiento estratégico, además de ser un reflejo de la capacidad de adaptación a las expectativas y necesidades cambiantes de su mercado actual y futuro. Este tipo de alumnos, claramente poseerían una cultura de Star-Up, asumiendo los riesgos necesarios para capturar el potencial de sus emprendimientos innovadores.

En esta investigación se realizó el análisis de las capacidades emprendedoras potenciales $y$ efectivas en alumnos de centros de educación superior, sin embargo en un futuro se espera analizar la influencia de los ecosistemas transformadores internos y externos en la evolución de esta capacidad emprendedora.

\section{Bibliografía}

ALCARAZ RODRIGUEZ, R. E. (2004). Efectividad del Curso de Emprendedores, en el desarrollo del perfil emprendedor del alumno. Monterrey.

ALFARO, J. R., y Gómez, J. D. (1999). Perfil del emprendedor exitoso en Venezuela. Caracas, Venezuela.: Instituto de Estudios Superiores de Administración, IESA.

ALVAREZ, R., y Jung, D. (2004). Educational curricula and self-efficacy: entrepreneurial orientation and new venture intentions among university students in Mexico. Frontiers of Entrepreneurship research .

AMORÓS,J. E., \& Poblete Cazenave, C. (2010). GEM Reporte Nacional de Chile. Global Entrepreneurship Monitor.

AYUGA TÉLLEZ, E., González García, C., Grande Ortiz, M., \& Martínez Falero, E. (20I2). Diseño de un cuestionario para evaluar conocimientos básicos de estadística de estudiantes del último curso de ingeniería. Formación Universitaria , 21 32.

BAUTISTA GARCÍA,V., \& Mateos Moreno, D. (2012). Diseño y validación de un cuestionario de evaluación de la actitud hacia la música clásica del alumnado de educación secundaria a obligatoria. Revista Electrónica de Investigación y Docencia (REID) , |4|-|6|.

BILBAO, A., y Pachano, S. (Enero de 2002). CID HARVARD. http://www.cid.harvard.edu/archive/andes/documents/ workingpapers/razgosyactitudes/rasgos_actitudes_venezuela_competitiva.pdf [28-12-12]

BURNETT, D. (2000). The supply of Entrepreneurship and Economic Development. Sitio web de Technopreneurial. com: http://www.asiaentrepreneurshipjournal.com/AJESIIIIAdegbite.pdf [10-04-I2]

BUSENITZ, L.W. (1999). Differences between entrepreneurs and managers in large organizations: Biases and heuristics in strategic decision-making. Journal of Bussines Management, 9-30.

CANO, C., García, J., y Gea, A. (2003). Actitudes emprendedoras y creación de empresas en estudiantes universitarios. Universidad de Almería .

CANTILLON, R. (1755). Ensayo sobre la naturaleza del comercio en general. www.eumed.net/cursecon/economistas/textos/cantillon_Naturaleza.htm [10-04-12]

ISSN: 07 I8-2724. (http://www.jotmi.org) 
CROSS, B., y Travaglione, A. (2003). the untold store: is the entreprener of the $2 I$ st century defind by emotional inteligence? The International Journal of Organizational Analysis , $221-228$.

ESTUDIO HAY GROUP. (2000). What makes a great entrepreneur? Londres: Hay Group.

FILION, J.,y Gilles, R. (1996). Escuela de Altos Estudios Comerciales (HEC). Montreal, Canada: Librería Universitaria.

FORMICHELLA, L. M. (2004). El concepto emprendimiento y su relación con la educación, el empleo y el desarrollo local. Bueno Aires, Argentina.

GARZÓN , M. (2004). La innovación intraemprendedora liderada por los gerente de las Pymes. Universidad empresa, 100.

GONZÁLEZ ORTIZ, R, Zúñiga Álvarez, A (20II). Journal of Technology Management \& Innovation. Vol 6, No I (20I I)

GONZÁLEZ DÍAZ, L.; Vidaud Quintana, I. Factores para evaluar la viabilidad de proyectos de conservación de edificaciones esenciales, no productivas, en zonas sísmicas Ingeniería Revista Académica,Vol. I3, Núm. I, enero-abril, 2009, pp. 25-39

GRANADO DE LA ORDEN, S., Rodríguez Rieiro, C., OImedo Lucerón, M., Chacón García, A., Vigil Escribano , D., y Rodríguez Pérez, P. (2007). Diseño y validación de un cuestionario para evaluar la satisfacción de los pacientes atendidos en las consultas externas de un hospital de Madrid en 2006. Revista Española de Salud Pública , 637-645.

HATTEN, T. (1993). Student attitude toward entrepreneurship as affected by participation in small business institute program. Michigan: UMI Dissetation Services.

GOMEZ BARAHONA, H., Martín Cruz, N., Rodriguez Escudero,A. I.,y Saboia, F. (2005). ¿El emprendedor nace o se hace? Un análisis de los determinantes del espíritu emprendedor. $\mathrm{XVI}$ Spanish-Portuguese meeting of scientic management , 33-34.

KÖTTNER JAN, S. D. (2010). consistencia interna y $\nabla$ de Cronbach: Un comentario sobre Beeckman et al. (2010). Revista Internacional de los Estudios de Enfermería ,Volumen 47, número 7, páginas 926-928, julio de 2010.

KRAUSS, C. (2005). Las actitudes Emprededoras en los Estudiantes de la Universidad Católica del Uruguay. Revista FCE , 3-4.
KRUEGER , N., y Brazeal, D. (1994). Entrepreneurial potential and potential entrepreneurs. Entrepreneurship Theory and Practice , 91-104.

LAUKKANEN, M. (2000). Exploring alternative approaches in high-level entrepreneurship education: creating microchanisms for endogenous regional growth. Entrepreneurship \& Regional Development , 25-48.

LISERAS , N., Guerrero de Rearte,A., y Graña, F. (2003). Factores asociados a la vocación emprendedora en alumnos universitarios. Universidad Nacional de Mar del Plata. Red Pymes.

LOLI A., Dextre E., Del Carpio J., La Jara E. (20I0).Actitudes de creatividad y emprendimiento en estudiantes de la universidad nacional de ingeniería y su relación con algunas variales socio demográficas. Perú: Universidad Nacional Mayor de San Marcos.

LÓPEZ PUMAR, G., del Castillo Martín , N., y Oramas Viera, A. (20II). Validez y confiabilidad del cuestionario Índice de Capacidad de Trabajo (ICT) en su versión cubana. Revista Cubana de Salud y Trabajo , 29-34.

LUCERO, I., y Meza, S. (2002). Validación de instrumentos para medir conocimientos. FACENA: Facultad de Ciencias Exactas y Naturales y Agrimesura de la UNNE .

MALLERY, G. (1995). SPSS: Step by Step A simply guide and reference. Estados Unidos: Belmont WADSWORTH.

MC CARTHY, B. (2003). The impact of the entrepreneur's érsonality on the strategy-formation and planning process in SMEs. Iris Journal of Management , 24, I 54.

MC CLELLAND, D. C. (196I). The Achieving of Management. NJ:Van Nostrand.

MORALES PARRAGUÉ,M.(2009). El clima de emprendimiento, un determinante clave en la intención emprendedora de los estudiantes de Escuelas de Negocio. Universidad de Chile. Santiago: Momento Cero.

MORALESVALLEJOS, P. (I7 de Diciembre de 20I2). El Análisis Factorial en la construcción e interpretación de test, escalas y cuestionarios. de Universidad Pontificia: http://www. upcomillas.es/personal/peter/investigacion/AnalisisFactorial. pdf [27-12-12]

NECK, C., Neck, H., Manz, C., y Godwin, J. (1999). I think I can I think I can. Journal of Managerial Psychology, I 4 (5/6), 477-50I. 
OSORIO GÓMEZ, J. C., y Orejuela Cabrera, J. P. (2008). EI proceso de análisis jerárquico (AHP) y la toma de decisiones multicriterio. Ejemplo de aplicación. Scientia et Technica , 248.

PALACIOS, L. E. (1999). Sabiduria popular en la empresa venezolana. Caracas, Venezuela: Universidad Católica Andrés Bello.

ROBINSON, P. (199I). An attitude approach to the prediction of entrepreneurship. Entrepreneurship:Theory \& Practice , 13.

RUIZ ARROYO, M., Bojica, A., Albacete Saez, C., y Fuentes Fuentes, M. (2008-10). ¿Como identifican las oportunidades de negocio los emprendedores? Una aproximación descriptiva al caso Andaluz. Asociación Española de Contabilidad y Administración de Empresas (AECA) , I I-23.

SCHUMPETER, J. A. (1942). Capitalismo, socialismo y democracia. Estados Unidos: Gerorge Allen y Unwin.

SORIA BARRETO, K. (2009-20I0). GEM, Informe De La Región de Coquimbo. Coquimbo: Universidad Catolica Del Norte.

STEWART,W. H., y Roth, P. L. (200I). Risk propensity difference between entrepreneurs and manager: $A$ meta- analytic review. Journal of Applied Psychology, I 45-153.

TIMMOS, J. (1985). New Venture Creation. USA: Ed. Irwin.

VANDER WERF, P., y C. Brush. (1989). Toward agreement on the focus of entrepreneurship research: progress without definition. USA: National Academy of Management.

VICENS, L., y Grullón, S. (20I I). Innovación y emprendimiento: Un modelo basado en el desarrollo del emprendedor. $\checkmark$ Foro de Competitividad de las Américas para el Banco Interamericano de Desarrollo y el Compete Caribbean (pág. II). Santo Domingo: Compete Caribbean.

\section{Agradecimientos}

Los autores agradecen el apoyo de la Universidad de La Serena, el Gobierno Regional de Coquimbo y de la Corporación de Fomento de la Producción (Chile), para la realización de este trabajo. 